\title{
AMBIDESTRIA ORGANIZACIONAL: UMA ANÁLISE DO ESTADO- DA-ARTE NA LITERATURA NACIONAL E INTERNACIONAL
}

Hélio Henrique de Matos (helio.hmatos@hotmail.com) - Instituto de Ciências Biológicas, Universidade Federal de Minas Gerais.

Franciele Garcia da Silva (franciele.ufmg@gmail.com) - Escola de Engenharia, Universidade Federal de Minas Gerais.

Tiago Paz Lasmar (tplasmar@gmail.com) - Escola de Engenharia, Universidade Federal de Minas Gerais.

Ana Valéria Carneiro Dias (anaval@ufmg.br) - Escola de Engenharia, Universidade Federal de Minas Gerais.

\begin{abstract}
RESUMO
O conceito de Ambidestria Organizacional (AO) procura conciliar as atividades de inovação incremental e radical nas organizações, propondo práticas organizacionais que favoreçam ambas as perspectivas. Organizações ambidestras seriam aquelas capazes de explorar simultaneamente as competências existentes e assegurar a viabilidade atual, sem deixar de fora novas oportunidades. $O$ debate sobre AO tem se tornado mais complexo à medida que vem se desenvolvendo, justificando a investigação do estado-da-arte do conceito. Este artigo apresenta um estudo bibliográfico acerca do tema $A O$, por meio de revisão sistemática de literatura, abrangendo periódicos internacionais $e$ nacionais. A amostra final teve um total de 74 artigos, analisados segundo ano de publicação, periódico, metodologia utilizada, nível de análise e tema central dos artigos. Buscou-se mapear quais são as principais definições e contribuições desse conceito, os principais autores e novas pesquisas da área. Observou-se um crescimento nas publicações na última década, principalmente de pesquisas empíricas, contudo, os estudos de ambidestria ainda debatem a definição conceitual e o entendimento teórico do fenômeno. Ressalta-se a identificação de somente quatro trabalhos no contexto brasileiro sobre o tema, o que expõe uma lacuna na literatura local e justifica a importância da elaboração de pesquisas, principalmente voltadas ao cenário produtivo nacional.
\end{abstract}

Palavras-chave: Ambidestria Organizacional, inovação radical, inovação incremental, exploration, exploitation

Área: Organização e gestão do conhecimento para o desenvolvimento de produtos

\section{INTRODUÇÃ̃O}

O tema inovação encontra-se difundido em várias definições, práticas e significados, sendo importante compreender o contexto de sua aplicação, possibilitando a evolução tanto conceitual quanto prática desse objeto de estudo (GOPALAKRISHNAN e DAMANPOUR, 1997). Uma categorização possível da inovação se dá de acordo com seus graus de intensidade, tais como incremental ou radical e/ou outros possíveis níveis intermediários (GRIFFIN e PAGE, 1996; TIDD, BESSANT e PAVITT, 2008). A inovação incremental tem como base a exploração do conhecimento existente na organização, enquanto a radical é fundamentada na criação de novos conhecimentos. Essa perspectiva está alinhada à definição de exploitation e exploration proposta por March (1991), que define exploitation como sendo 
todas as atividades que englobam o refinamento, escolha, produção, eficiência, seleção, implementação e execução de um produto/serviço. Já atividades de exploration são descritas por pesquisa, variação, tomada de risco, experimentação, flexibilidade, e descoberta. $\mathrm{O}$ autor ainda ressalta que manter o equilíbrio adequado entre elas é o fator primordial para a sobrevivência e prosperidade do sistema.

Tidd, Bessant e Pavitt(2008) definem a inovação como o processo de transformar ideias em realidade e lhes capturar o valor, devendo ser gerida para que ocorra de forma sistêmica em uma organização. A partir dessa definição e da existência de diferentes níveis de inovação surgem perguntas como: quais são as práticas de gestão para se alcançar a inovação? Essas práticas se diferem de acordo com o nível de inovação que se deseja? É possível alinhar as práticas para os objetivos de curto e longo prazo e para os desenvolvimentos de baixo e alto risco?

Essas questões embasam o estudo do conceito de ambidestria organizacional (AO), que parte do pressuposto de que é possível que uma organização inove de maneira incremental e radical, buscando entender quais são as práticas e estruturas organizacionais que favorecem as inovações nessas diferentes perspectivas. As organizações ambidestras seriam aquelas capazes de explorar simultaneamente as competências existentes e assegurar tanto a viabilidade atual, sem deixar de fora novas oportunidades, como a futura. (MARCH, 1991; LEVINTHAL, 1993; O’REILLY III e TUSHMAN, 2004; RAISCH et al., 2009).

Apesar do aumento de publicações deste tema, devido à variedade de domínios de investigação, o debate inicial e principal sobre AO tem se tornado cada vez mais desconectado e complexo, o que justifica o desenvolvimento de trabalhos cujo objetivo é entender o estado da arte desse objeto de estudo (RAISCH e BIRKINSHAW, 2008).

O objetivo deste artigo é expor um estudo bibliográfico acerca do tema $\mathrm{AO}$, por meio de revisão sistemática de literatura, abrangendo periódicos internacionais e nacionais. Buscou-se mapear quais são as principais definições e contribuições desse tema, além de identificar quais os principais autores, novas pesquisas da área e possíveis hipóteses que os dados possam fornecer sobre a temática. $\mathrm{O}$ artigo está organizado, em sequência, pela apresentação da metodologia, onde serão explicitadas as etapas do trabalho, seguido dos resultados, com a exposição dos conceitos identificados e a análise dos dados obtidos; por fim, são apresentadas as conclusões da pesquisa e limitações.

\section{METODOLOGIA}

A metodologia adotada foi a revisão sistemática. Para Bereton et al. (2005) por meio de uma revisão sistemática, é possível que o pesquisador obtenha uma avaliação criteriosa e confiável das pesquisas realizadas dentro de uma temática específica. Seu processo é composto por coletar, conhecer, compreender, analisar, sintetizar e avaliar um conjunto de artigos científicos com o propósito de criar um embasamento teórico-científico (estado da arte) sobre um determinado tópico ou assunto pesquisado (KITCHENHAM, 2004).

O levantamento de dados se iniciou com a definição das palavras-chave e das bases de dados a serem pesquisadas. As palavras chaves utilizadas foram: Ambidestria, Ambidestria Organizacional, Exploração, Explotação, Ambidexterity, Organizational ambidexterity, Exploration e Exploitation, Eficiência e Inovação. A escolha das palavras chaves buscou englobar não só artigos que usassem o termo ambidestria, mas também aqueles que abordassem o tema de maneira geral, como por exemplo, artigos que tratassem da relação 
entre eficiência e inovação, que é um tópico relevante no assunto, e que não seria um resultado da pesquisa se apenas o termo ambidestria tivesse sido utilizado. Foram escolhidas as bases Google Acadêmico, Scielo e Web of Knowledge. Essa escolha baseou-se principalmente pela intenção de se encontrar artigos das principais revistas no cenário mundial, mas também trabalhos desenvolvidos no contexto brasileiro. A pesquisa ainda contou com um filtro por ano, considerado o período de 1991 até 2016, tendo como 1991 como marco inicial da pesquisa devido ao trabalho de March (1991) considerado pioneiro no estudo do tema.

A primeira etapa da pesquisa retornou 8917 resultados, grande parte deles desalinhados às propostas e objetivos do trabalho. Dessa maneira, os artigos encontrados foram filtrados por título, resultando um total de 272 artigos. Foram removidos principalmente artigos da área médica e relacionados com práticas de Recursos Humanos e Marketing que não estavam diretamente relacionados com os desafios das organizações de lidar com o tema ambidestria.

Na segunda etapa, foi feita a leitura dos resumos dos artigos selecionados, possibilitando uma avaliação mais aprofundada do objeto de estudo do artigo. A partir dessa análise, conseguiuse distinguir as metodologias utilizadas pelos autores, principais objetivos dos textos e os conceitos de ambidestria utilizados. Mais uma vez buscou-se filtrar os artigos que estavam mais alinhados com os objetivos de pesquisas propostos. Como resultado da segunda etapa obteve-se 87 artigos para serem lidos na íntegra, contudo não se obteve acesso ao texto em 13 dos casos, e a amostra foi reduzida para um total de 74 artigos para leitura.

\section{RESULTADOS}

Como resultado da revisão bibliográfica realizada, elaborou-se um quadro com as informações sobre o ano de publicação, periódico, método utilizado, nível de análise e o tema central dos artigos selecionados, informações estas apresentadas separadamente no decorrer dos resultados.

Embora a AO seja um tema relativamente incipiente, seu conceito e paradigmas têm sido cada vez mais discutidos e recebido contribuições de diversos fluxos de pesquisa, melhorando o entendimento sobre o fenômeno, mas também tornando sua literatura bastante fragmentada (NOSELLA, CANTARELLO e FILIPPINI, 2012). Nesse sentido, foi realizado um mapeamento dos principais conceitos utilizados nos artigos analisados, visando identificar as principais definições da $\mathrm{AO}$, bem como melhorar entendimento sobre a temática. A partir disso, os principais achados são apresentados abaixo como um apanhado geral sobre o tema.

\subsection{Ambidestria organizacional: conceitos e definições}

Os estudos considerados pioneiros na definição de ambidestria são os de Duncan (1976) e March (1991). Para March (1991), um fator primordial na sobrevivência e prosperidade dos sistemas de produção é a manutenção do equilíbrio entre exploration e exploitation. A explotação ou exploitation tem seu foco voltado ao desenvolvimento de atividades e estratégias relacionadas à eficiência, otimização e implementação, o que pode melhorar o desempenho em curto prazo, mas também representar um desafio à organização, frente a responder adequadamente às mudanças ambientais (MARCH, 1991; AHUJA e LAMPERT, 2001). Já a exploração ou exploration volta-se a pesquisa, assumir riscos, flexibilidade e inovações de cunho radical, que podem propiciar novos conhecimentos e direcionamentos, mas também representar um ciclo interminável de busca e mudança não recompensadora e com grande dispêndio de capital (MARCH, 1991; KANE; ALAVI, 2007). 
March (1991) ainda destaca que tais atividades demandam estratégias, contextos e estrutura organizacional diferentes e ressalta que o grande desafio, é alinhar a capacidade de uma organização explorar novos negócios, e, ao mesmo tempo, valer-se das competências já existentes.

Em 1996, Tushman e O'Reilly afirmam que a AO está na capacidade de se obter vantagem competitiva por meio da aptidão de alinhamento em sua operação corrente e adaptabilidade às mudanças no ambiente a qual a empresa está inserida, mas especificamente como sendo a capacidade de simultaneamente desenvolver inovação incremental e desruptiva na empresa. Os autores sugerem que um desempenho superior é esperado das organizações ambidestras, para tanto, ressaltam a importância de aspectos como estratégia, cultura e processos, e descrevem mecanismos estruturais para propiciar tal comportamento, destacando a criação de unidades autônomas de negócio como a principal forma de viabilização.

Pesquisadores sobre este tema têm observado diferentes formas com que as empresas praticam a ambidestria. A primeira delas é chamada "separação estrutural", onde existe a divisão entre exploration e exploitation em unidades organizacionais estruturalmente e espacialmente distintas (TUSHMAN e O'REILLY, 1996; BENNER e TUSHMAN 2003). Deste modo, cada unidade tende a apresentar características mais condizentes aos seus objetivos e ambientes onde estão inseridas (LAWRENCE e LORSCH, 1967). As unidades que desenvolvem exploration tendem a ser menores, mais flexíveis e descentralizadas, opostamente às centradas em exploitation (BENNER e TUSHMAN 2003, TUSHMAN e O'REILLY, 1996).

Alternativa à separação estrutural, as denominadas "estruturas paralelas" são uma abordagem onde se tem exploration e exploitation dentro da mesma unidade espacial, permitindo assim que as organizações e as pessoas alternem entre as duas estruturas organizacionais conforme as necessidades de exploração ou explotação, além de solucionar o problema de um possível distanciamento que possa ocorrer em unidades estruturalmente separadas (RAISCH e BIRKINSHAW, 2008; DEVINS e KÄHR, 2010).

A abordagem da integração ou ambidestria contextual baseia-se em mecanismos comportamentais para superar as tensões e viabilizar dentro de uma mesma unidade e estrutura organizacional as atividades de exploração e explotação (RAISH et al., 2009). Segundo Gibson e Birkinshaw (2004), a ambidestria está ligada majoritariamente a atividades gerenciais, portanto, pode ser construída dentro de uma organização e considerada uma capacidade organizacional. No entanto, segundo os autores, para que ocorra a integração, é necessário tanto um alinhamento entre os funcionários e a alta gerência, como também a adaptabilidade às demandas ambientais. Essa perspectiva de ambidestria ressalta a importância do indivíduo, que a partir do contexto organizacional o qual está inserido, passa a tomar a decisão sobre como dividir seu tempo entre demandas conflitantes de alinhamento e adaptabilidade.

Por fim, a abordagem do balanceamento temporal, como o próprio nome sugere, trata-se de uma separação temporal entre fases de exploration e exploitation, que pode partir de demandas externas, fatores situacionais, ou mesmo de acordo com a estratégia da organização (JANSEN et al., 2005). Ainda segundo o autor, o comportamento das organizações conforme explicado acima pode ser considerado dinâmico, no entanto, as organizações podem apresentar uma configuração estática com o passar do tempo, com pré-definições das proporções de atividades e projetos dedicados exploration e exploitation, por exemplo.

\subsection{Dispersão das publicações no tempo}


As demais informações compiladas a partir dos artigos analisados oferecem indicativos e hipóteses que compõem a realidade das pesquisas em AO. Estes resultados são apresentados e ilustrados nas seções seguintes.

A distribuição dos artigos nas quase três décadas analisadas, ilustrada na Tabela 1, demonstra que, embora a AO tenha sua primeira grande referência com March (1991) e, portanto, 25 anos até o ano de 2016, existiram muitos lapsos de tempo onde a temática não foi discutida, ou foi em pequena escala e sem grandes contribuições. Somente em 2004, treze anos após o artigo de March (1991), e oito anos após o de Tushman e O'Reilly (1996), novas contribuições foram feitas, no entanto, nota-se um real crescimento somente após o ano de 2008 .

Tais informações evidenciam que, apesar de já ter mais de 25 anos desde a primeira grande contribuição, o debate sobre a AO ficou dormente por muitos anos, o que poderia justificar a incipiência da temática em questões de definição do conceito e do próprio entendimento do fenômeno.

Tabela 1 - Número de publicações por

\begin{tabular}{|c|c|c|c|c|c|c|c|c|c|c|c|c|c|c|}
\hline ANO & 1991 & 1996 & 2004 & 2005 & 2007 & 2008 & 2009 & 2010 & 2011 & 2012 & 2013 & 2014 & 2015 & 2016 \\
\hline №́de publicações & 1 & 1 & 3 & 1 & 1 & 5 & 13 & 6 & 2 & 5 & 6 & 9 & 8 & 13 \\
\hline
\end{tabular}

Fonte: Elaborada pelos autores (2017)

\subsection{Número de artigos por periódico}

Tendo em vista o crescimento das publicações e o próprio impacto e qualidade destas, foram mapeados os periódicos de origem dos artigos selecionados. Nota-se que os periódicos com maiores números de artigos publicados sobre a temática AO são o Organization Science (JCR 3,360; 13 publicações) e o Journal of Management Studies (JCR 4,260; 5 publicações). O perfil das revistas, área de gestão e organização, corrobora com o fato de que o conceito de ambidestria é utilizado em sua maior amplitude para uma demanda de gestão organizacional, não necessariamente vinculado ao tema gestão da inovação. Trata-se então de um conceito utilizado para gestão de visões conflitantes dentro de uma organização.

\subsection{Metodologias utilizadas nos artigos}

Voltando o olhar para a classificação das metodologias dos artigos analisados, observou-se uma preponderância para os surveys e estudos de caso, conforme exposto na Figura 1.

Figura 1 - Número de artigos por metodologia

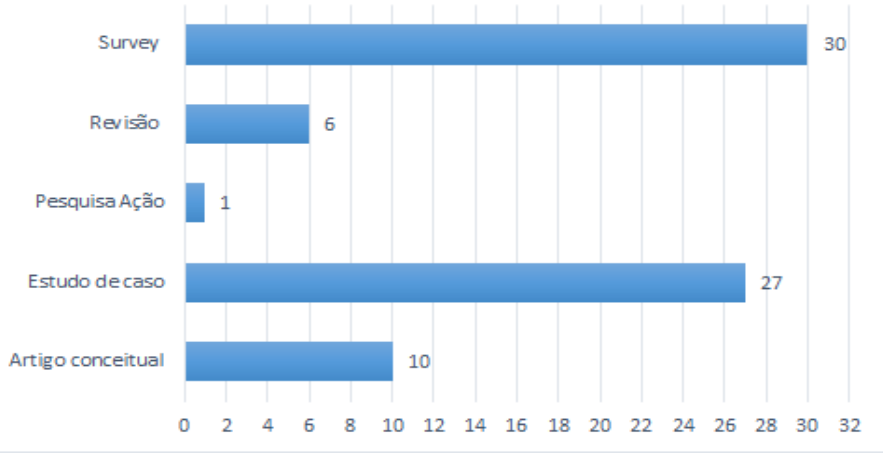

Fonte: Elaborada pelos autores (2017) 
O alto número de estudos com caráter empírico traz à tona algumas hipóteses e observações. A primeira delas é a indicação do interesse, ou mesmo a necessidade de se estudar e entender o comportamento ambidestro in loco, dada a complexidade do fenômeno e nebulosidade de seus conceitos. A segunda hipótese diz respeito do interesse prático das empresas pela temática, tendo em vista as grandes amostras de respondentes, bem como uma possível imprescindibilidade de resolução dos conflitos gerados pela ambidestria na prática.

Acerca do uso da metodologia survey, entende-se a necessidade da realização de pesquisas exploratórias a fim de se identificar conceitos principais, descobrir novas dimensões e entender a organização das empresas para com a ambidestria. De forma semelhante, os estudos de casos buscam analisar a realidade e as práticas das empresas, porém, enquanto os surveys objetivam o entendimento a partir de um grande número de respondentes, os estudos de casos preocupam-se em avaliar com profundidade as unidades de análise e tirar conclusões a partir dos comportamentos observados.

\subsection{Nível de análise privilegiado}

Outra característica importante dos artigos selecionados para esta pesquisa foi o nível de análise observado e tratado pelos trabalhos. Obteve-se o seguinte panorama: (I) nível do indivíduo ou operacional: 9 artigos; (II) nível da equipe ou tático: 5 artigos; (III) nível da organização ou estratégico: 67 artigos; e (IV) nível de projeto: 4 artigos.

De fato, os artigos foram selecionados priorizando aqueles que traziam a visão de ambidestria sob a perspectiva da organização, no entanto, muitos destes trabalhos apresentavam uma análise multinível, trazendo abordagens sob a perspectiva do indivíduo e/ou equipe e/ou projeto. Isso faz sentido se pensarmos nas quatro abordagens anteriormente expostas: separação estrutural; estruturas paralelas; balanceamento temporal; e abordagem contextual, que buscam a ambidestria a partir de diferentes aspectos na instituição, mas com o objetivo final comum de beneficiar a organização como um todo. Além disso, com exceção da contextual, as demais abordagens concentram-se em estruturas e estratégias em nível da empresa, com processos decisórios de responsabilidade da alta gerência. Contudo, o nível do indivíduo é destacado em alguns artigos, principalmente como elemento chave para o desenvolvimento da abordagem contextual da ambidestria, o que justifica a presença de trabalhos com desenvolvimento sob esse nível de análise.

\subsection{Perspectivas de aplicação da ambidestria}

Por fim, têm-se as áreas de gestão nas quais a ambidestria se aplica abordadas em cada artigo, a saber: (a) empreendedorismo; (b) gestão estratégica; (c) gestão da inovação; (d) design organizacional; (e) desempenho organizacional; (f) aprendizagem organizacional e; (g) antecedentes da AO.

Conforme exposto na Figura 2, é possível notar que os artigos encontram-se dispostos em diversas temáticas, o que confirma a constatação de Raisch e Birkinshaw (2008) a respeito da grande dispersão da AO entre várias áreas, ainda atualmente. Por outro lado, tal dispersão poderia identificar a relevância e envolvimento de diversas áreas de pesquisa e também das próprias organizações no comportamento ambidestro.

A perspectiva do empreendedorismo é abordada dentro da temática da $\mathrm{AO}$, principalmente, por meio de iniciativas de intraempreendedorismo como atividades de exploration, buscando a criação de novos empreendimentos, modelos de negócios e renovação organizacional. Vale 
a pena ressaltar tal achado, uma vez que, diferente das demais áreas, a interrelação entre a AO e o empreendedorismo pode não ser trivial.

Figura 2 - Áreas de gestão abordadas nos artigos

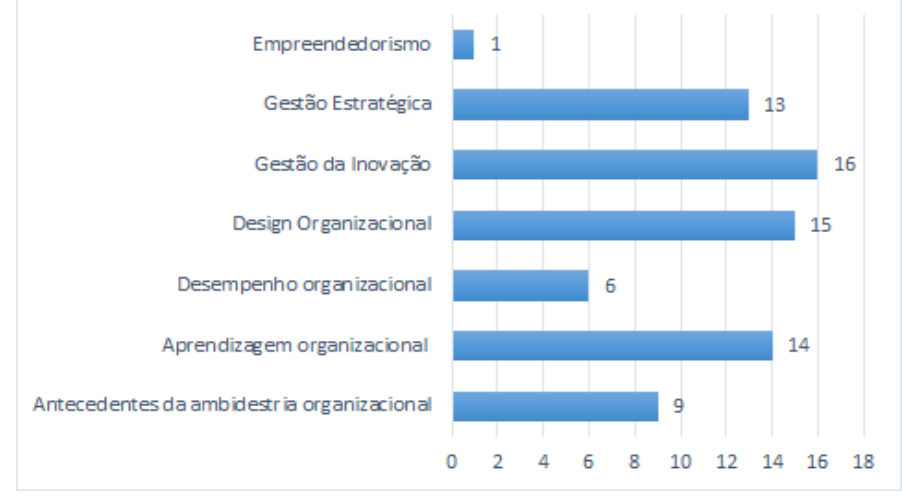

Fonte: Elaborada pelos autores (2017)

\subsection{O contexto brasileiro de pesquisas sobre Ambidestria Organizacional}

Do total de artigos selecionados apenas quatro foram estudos no Brasil. O primeiro deles, Martins et al (2014), apresenta um survey, aplicado junto às empresas vinícolas brasileiras. $\mathrm{O}$ artigo busca fazer uma correlação entre os diferentes tipos de comportamento estratégico dos gestores das vinícolas nacionais com o processo de AO. O segundo artigo, de Karrer et al (2015), é um trabalho conceitual que apresenta as atividades de exploration e exploitation como paradoxais, e quatro estados de $\mathrm{AO}$, baseados em táticas de separação e integração são discutidos: organizações ambidestras de longo e curto prazo, organização não estruturada para ambidestria e organização monolítica, focada apenas nas táticas integrativas.

Já o trabalho de Bandeira-de-Mello et al.(2015)analisa a expansão de multinacionais de países emergentes para outros países, sob a perspectiva da ambidestria. Essas organizações têm que explorar simultaneamente novas capacidades para atuação em novos países, mantendo o desempenho em seus países de origem. O estudo de caso em uma empresa brasileira faz a análise por meio de três variáveis-chave: modo de operação, estrutura organizacional e competição por recursos. Por fim, o trabalho de Dunlap et al. (2016) analisa, no contexto brasileiro, como a aquisição e utilização de conhecimento externo, seja por meio de um maior envolvimento do fornecedor ou absorvendo conhecimento de multinacionais, impacta diretamente no desafio da ambidestria.

\section{CONSIDERAÇÕES FINAIS}

O presente artigo teve como objetivo apresentar um estudo bibliográfico, visando um maior entendimento acerca do tema $\mathrm{AO}$ e da forma como ele tem sido discutido em diferentes áreas. As pesquisas referentes à $\mathrm{AO}$ têm ganhado destaque nos últimos anos, principalmente as de caráter empírico, ainda que questões conceituais continuem abertas. Percebeu-se que os trabalhos apresentam características exploratórias pelos tipos de metodologias utilizadas e grande variedades de temas em que esse objeto de estudo é abordado. Os resultados e análises também sugeriram que a ambidestria é tratada principalmente no nível estratégico das organizações, e que surgiu como uma abordagem de gestão empresarial, posteriormente apropriada pela gestão da inovação.

A identificação e análise do modo como o tema ambidestria tem sido discutido tornou possível compreender os gaps existentes com relação ao conteúdo, bem como servirá como 
embasamento para a realização de pesquisas futuras. No Brasil, o tema ainda não apresenta recorrência de publicação em revistas, o que expõe uma lacuna na literatura local e justifica a importância de estudos como este, no intuito de ampliar e incentivar o debate.

Apesar da abrangência das bases de dados consultadas, sendo duas delas internacionais e uma latino-americana, o artigo apresenta como limitação a ausência de consulta em demais bases de dados existentes, tais como banco de teses das universidades nacionais, e de congressos. Além da falta de acesso a alguns artigos que retornaram nas pesquisas realizadas e, apesar de terem potencial contribuição para a pesquisa, não puderam ser utilizados. Espera-se preencher tais lacunas com a continuação das pesquisas sobre o tema.

\section{REFERÊNCIAS}

AHUJA, Gautam; MORRIS LAMPERT, Curba. Entrepreneurship in the large corporation: A longitudinal study of how established firms create breakthrough inventions. Strategic Management Journal, v. 22, n. 6-7, p. 521-543, 2001.

BANDEIRA-DE-MELLO, Rodrigo et al. Unpacking the ambidexterity implementation process in the internationalization of emerging market multinationals. Journal of Business Research, v. 69, n. 6, p. 2005-2017, 2016.

BENNER, Mary J.; TUSHMAN, Michael L. Exploitation, exploration, and process management: The productivity dilemma revisited. Academy of management review, v. 28, n. 2, p. 238-256, 2003.

BERETON et al. Lessons from Applying the Sistematic Literature Review Process within the Software Engineering Domain. The Journal of System and Software, v. 80, p.571-583, 2007.

BIRKINSHAW, Julian; GIBSON, Cristina.Building ambidexterity into an organization. MIT Sloan management review, v. 45, n. 4, p. 47, 2004.

DEVINS, Gaëtan; KÄHR, Caroline N. Structuring ambidextrous organizations: Exploitation and exploration as a key for long-term success. More than bricks in the wall:

Organizational perspectives for sustainable success, p. 60-67, 2010.

DUNLAP, Denise et al. Organizing for Innovation Ambidexterity in Emerging Markets Taking Advantage of Supplier Involvement and Foreignness. Journal of Leadership \& Organizational Studies, 2016.

GIBSON, Cristina B.; BIRKINSHAW, Julian.The antecedents, consequences, and mediating role of organizational ambidexterity. Academy of management Journal, v. 47, n. 2, p. 209226, 2004.

GOPALAKRISHNAN, S.; DAMANPOUR, F. a Review of innovation research in Economics, Sociology and Technology Management. Omega, International Journal of Management Science., Vol. 25, n.1, p. 15-28, 1997.

GRIFFIN, Abbie; PAGE, Albert L. PDMA success measurement project: recommended measures for product development success and failure. Journal of product innovation management, v. 13, n. 6, p. 478-496, 1996.

JANSEN J.J.P., VAN DEN BOSCH F.A.J. and VOLBERDA H.W. Exploratory innovation, exploitative innovation, and ambidexterity: The impact of environmental and organizational antecedents. Schmalenbach Business Review (SBR), vol. 57(4): p. 351-363, 2005. 
KANE, Gerald C.; ALAVI, Maryam. Information technology and organizational learning: An investigation of exploration and exploitation processes. Organization Science, v. 18, n. 5, p. 796-812, 2007.

KARRER, Daniel; FLECK, Denise.Organizing for Ambidexterity: A Paradox-based Typology of Ambidexterity-related Organizational States. BAR-Brazilian Administration Review, v. 12, n. 4, p. 365-383, 2015.

KITCHENHAM, B. Procedures for performing systematic reviews, Joint Technical Report Software Engineering Group, Department of Computer Science, Keele University, United King and Empirical Software Engineering, National ICT Australia Ltd., Australia, 2004.

LAWRENCE, Paul R.; LORSCH, Jay W. Differentiation and integration in complex organizations. Administrative science quarterly, p. 1-47, 1967.

LEVINTHAL, D., MARCH, J. Myopia of learning. Strategic Management Journal, 14: 95-112. 1993.

MARCH, James G. Exploration and exploitation in organizational learning. Organization science, v. 2, n. 1, p. 71-87, 1991.

MARTINS, Elvis Silveira et al.Comportamento estratégico e ambidestria: um estudo aplicado junto às empresas vinícolas brasileiras. Revista Brasileira de Gestão de Negócios, v. 16, n. 52, p. 392, 2014.

NOSELLA, Anna; CANTARELLO, Silvia; FILIPPINI, Roberto. The intellectual structure of organizational ambidexterity: A bibliographic investigation into the state of the art. Strategic Organization, v. 10, n. 4, p. 450-465, 2012.

O'REILLY, Charles A.; TUSHMAN, Michael L.The ambidextrous organization. Harvard business review, v. 82, n. 4, p. 74-83, 2004.

RAISCH, Sebastian; BIRKINSHAW, Julian. Organizational ambidexterity: Antecedents, outcomes, and moderators. Journal of management, 2008.

RAISCH, Sebastian et al. Organizational ambidexterity: Balancing exploitation and exploration for sustained performance. Organization science, v. 20, n. 4, p. 685-695, 2009. TIDD, J.; BESSANT, J.; PAVITT, K. Gestão da inovação. 3 ed. São Paulo: Bookman, 2008. TUSHMAN, Michael L.; O'REILLY, Charles A. The ambidextrous organizations: Managing evolutionary and revolutionary change. California management review, v. 38, p. 8-30, 1996. 